\title{
MATRIMONIO IGUALITARIO Y PARENTESCO: DISCURSOS, HEGEMONÍAS Y ACONTECIMIENTO
}

\author{
Analía BuZaglo ${ }^{1}$; Mariela Morandi² y Mónica Culla ${ }^{3}$ \\ Universidad Nacional de Rosario y Municipalidad de Rosario. \\ Argentina
}

Recibido/12/03/2012

Aceptado/24/05/2012

\section{Resumen}

La sanción de la ley de matrimonio igualitario en Argentina, el 15 de julio de 2010, en tanto acontecimiento cultural, no sólo ha visibilizado realidades diversas existentes hace tiempo sino que ha permitido dar a conocer y debatir cuánto y cómo estas realidades están siendo alojadas en nuestra sociedad y en sus instituciones. En el presente artículo pondremos el foco en la homoparentalidad, en tanto praxis social que interpela saberes hegemónicos acerca de las nociones de familia, filiación y de parentesco. Realizaremos un recorrido por diferentes autoras/es que examinan críticamente las formas hegemónicas de comprender estas nociones. Asimismo aportaremos una lectura específica desde la perspectiva de los Derechos Humanos y los movimientos sociales.

Palabras clave: familia, matrimonio igualitario, diversidad sexual, parentesco, homoparentalidad.

1. Master en Investigación en Psicología Social por la Universidad Autónoma de Barcelona (UAB). Diploma de Estudios Avanzados en Investigación Social (DEA). Docente e investigadora de la Facultad de Psicología de la Universidad Nacional de Rosario (UNR)

2. Magíster en Ciencias Sociales. Trabajadora Social. Área de Diversidad Sexual - Secretaría de Promoción Social - Municipalidad de Rosario. Docente integrante del Núcleo de Estudios y Extensión en Género de la Escuela de Trabajo Social (UNR)

3. Psicóloga. Área de Diversidad Sexual - Secretaría de Promoción Social - Municipalidad de Rosario. Trabajo clínico. 


\begin{abstract}
The Law for Equal Marriage passage in Argentina on July the 15th 2010, was not just a cultural event. Not only has it helped to visualise different existing realities from a long time ago but it has also helped to promote the debate about how and how deep these realities are held in our society and its institutions. In this paper we will concentrate in the homoparentality as a social praxis that questions hegemonic notions about family, filiation and kinship. We will look at different authors that critically examine the hegemonic ways of understanding these concepts. We will also provide a specific perspective from Human Rights and social movements.
\end{abstract}

Keywords: family, equal marriage, sexual diversity, kinship, homoparentality. 


\section{Introducción}

El estudio que fundamenta este artículo, surge a partir de la motivación de tres profesionales que desde dos ámbitos diferentes -Universidad Nacional de Rosario y Área de Diversidad Sexual de la Municipalidad de Rosario- se vieron interpeladas en sus prácticas y saberes, por las realidades de las familias homoparentales.

Con la sanción de la Ley de Matrimonio Igualitario y los debates en torno a la media sanción de la Ley de Identidad de Género en Argentina, se visibilizaron realidades LGBTI en la sociedad argentina, generando un movimiento cultural y social que trascendió ampliamente el ámbito de las organizaciones de diversidad sexual.

Este acontecimiento cultural nos conduce al análisis de un universo complejo y en expansión: nuevas configuraciones vinculares y familiares, nuevas formas de nombrar roles y funciones, nuevas estrategias para enfrentar obstáculos institucionales cotidianos en el ejercicio de los derechos.

A partir de ello, consideramos importante ampliar el universo de saberes previos, donde aún coexisten prejuicios y paradigmas cristalizados, recreando conceptualizaciones y resignificando las ya existentes sobre la materia.

La importancia de esta investigación radica en su contribución a la visibilización de las nuevas configuraciones familiares habilitando la pregunta por las dificultades que las mismas enfrentan socialmente, sin olvidar los aportes que realizan al resto de la sociedad, en términos de instituir nuevas realidades o transformar las ya existentes.

\section{Matrimonio y parentesco: filiación y orden cultural}

Resulta claro que los debates sobre el matrimonio y el parentesco gay, dos temas que a menudo se juntan, se han convertido en sitios de un intenso desplazamiento de otros temores políticos, temores sobre la tecnología, sobre nuevas demografías y también sobre la unidad y transmisibilidad mismas de la nación, y temores de que el feminismo, al insistir en la atención infantil, 
haya logrado extender el parentesco fuera de la familia y lo haya abierto a los extraños. ${ }^{4}$

Asistimos a una crisis del concepto de familia nuclear en sociedades globalizadas, posindustriales, posmodernas y con una fuerte impronta heterocentrista. Las formas de organización familiar se diversifican cada vez más: la caída del padre de familia, la deconstrucción de la maternidad y el auge de las nuevas tecnologías reproductivas ponen en cuestión que la unión de varones y mujeres sea un elemento esencial para la procreación, desafiando entre otros, el concepto tradicional de parentalidad. Así, entran en relación y se visibilizan categorías como homosexualidad, y parentalidad.

Estas nuevas dinámicas sexuales, en tanto concebimos la diversidad sexual desde una perspectiva histórica y no esencialista, ponen en cuestión la naturalización de aspectos, que podríamos considerar fundadores, de las configuraciones culturales hegemónicas; y por ello mismo, son objeto de todo tipo de discriminación y exclusión. Al decir de Beatriz Preciado: «Los accidentes de género certifican el género como tecnología ${ }^{5}$, develando la nonaturalidad de la normatividad heterosexual compulsiva. ${ }^{6}$

En este sentido, en el marco de los debates en torno a la Ley de Matrimonio Igualitario en Argentina, pero también en países europeos, como Francia, se ha sostenido que esta Ley atenta contra el orden cultural y simbólico. De este modo, queda en evidencia que el binomio naturaleza-cultura -ya sea que la balanza caiga de un lado o del otro-, también constituye un artificio con el cual argumentar posiciones homófobas. Como ejemplo, dentro del campo del psicoanálisis y de la filosofía encontramos autoras/es que localizan el pasaje de la naturaleza a la cultura a partir de la relación heterosexual, dando cuenta de una lectura sesgada de los textos de Sigmund Freud y Jacques Lacan ${ }^{7}$.

En una entrevista con Jacqueline Rose, en 1990, la conocida kleiniana Hanna Segal reitera su opinión de que «la homosexualidad es un ataque a la pareja progenitora» y una «interrupción del desarrollo»; expresa su furia ante la situación en que dos lesbianas crían a un niño y agrega que considera que «la estructura homosexual adulta es patológica ${ }^{8}$

\footnotetext{
4. BUTLER, Judith. «Is kinship always already heterosexual», en Butler, Judith: Undoing gender, Nueva York, Routledge 2004, pp. 102-130.

5. <http://paroleparole-paroleparole.blogspot.com/2012/01/porque-hasta-que-todas-lasmujeres-sean.html> consultado el 23 de enero de 2012.

6. <http://www.pts.org.ar/spip.php?article15792> consultado el 30 de octubre de 2011.

7. <www.letraese.org.mx/orientacion.DOC> consultado el 25 de enero de 2012.

8. ButLer, Judith. Undoing gender. Routledge, Nueva York, 2004. pp. 32, 33.
} 
Podemos encontrar en algunas lecturas que se han realizado del texto antropológico Las estructuras elementales del parentesco, de Claude Levi-Strauss, de 1949, versiones limitadas de la problemática de la prohibición del incesto. En términos del análisis que hace Butler:

Para Lévi-Strauss, el drama edípico no debía concebirse como un momento o fase del desarrollo; por el contrario, consiste en una prohibición que está presente en el momento de iniciarse en el lenguaje, que a veces sirve para facilitar a todos los sujetos incipientes la transición de la naturaleza a la cultura. No es que con el tiempo se llegue a la barrera que prohíbe la unión sexual con la madre, sino que, en cierto sentido, se encuentra allí como una condición previa para la individuación, como un supuesto y un soporte para la inteligibilidad cultural misma. Ningún sujeto surge sin contar con esta barrera o prohibición como su condición, y no es posible reclamar ninguna inteligibilidad cultural sin pasar primero por esta estructura fundacional. Ciertamente, la madre queda vedada porque pertenece al padre, de modo que si esta prohibición es fundamental y comprendida, entonces el padre y la madre existen como aspectos lógicamente necesarios de la prohibición misma. Ahora bien, el psicoanálisis explica que no es necesario que el padre y la madre existan de verdad, pues pueden ser posiciones o figuras imaginarias, pero sí deben aparecer estructuralmente de alguna manera. ${ }^{9}$

Ahora bien, veamos de qué se trata esta construcción cultural heterosexual, anclada en la biología y en el «orden simbólico» y cuáles han sido sus bases, de modo que podamos comprender, contra qué atenta el matrimonio igualitario y sus formas de parentesco.

Dice Diana Maffía que las posiciones conservadoras respecto a la sexualidad se han sostenido en tres creencias básicas:

a) que los sexos son sólo dos: masculino y femenino, de tal forma que la dicotomía anatómica conlleva una dicotomía de géneros.

b) que el sexo es sólo para la reproducción, lo que implica que cualquier práctica sexual que no esté aplicada a la reproducción no es aceptada.

c) que la familia es una unidad natural, en la que hay algo que los sujetos pactan, organizan entre sí mediante un contrato voluntario pero que hay un núcleo que es como los átomos de esa configuración y ese átomo es la familia. La familia aparece entonces del lado de la naturaleza, con una lógica distinta a la política. ${ }^{10}$

En resumen, podemos enumerar estas bases diciendo que se trata de la dicotomización de los géneros en masculino y femenino; de la comprensión de la

9. Ibíd., pp. 22, 23.

10. MAFFIA, Diana. «Familia y adopción: reflexiones acerca de la homoparentalidad», en Rotenberg, E., Wainer Agrest, B. (comp.): Homoparentalidades. Nuevas familias. Bs. As. Lugar Editorial. 2007. pp. 57-62.

Feminismo/s 19, junio 2012, pp. 51-65 
sexualidad humana por la vía reproductiva y de la concepción de la familia en tanto «unidad natural».

Basta hacer un breve recorrido por los desarrollos contemporáneos en materia de Género -como los de la Teoría Queer ${ }^{11}$ - Sexualidad - Teoría sexual freudiana, antropología estructuralista, deconstruccionismo filosófico, estudios culturales, re-lecturas de la teoría psicoanalítica realizadas por teóricas feministas ${ }^{12}$, grupos de liberación homosexuales, movimiento feminista, etc. y Familia (entre otros, los actuales desarrollos antropológicos en materia de parentesco y familia ${ }^{13}$ ), para comprobar que estas bases constituyen verdaderas creencias, o al decir de Castoriadis ${ }^{14}$ : imaginarios sociales construidos en los entramados del Poder-Saber ${ }^{15}$.

Por tanto consideramos que, tal como enuncia Andrés Castelar ${ }^{16}$, la familia no es un edificio monolítico que se ve socavado y desafiado, sino un hecho social naturalizado que puede ocasionar nuevas relaciones entre sus miembros. Estas nuevas configuraciones abren, a su vez, nuevas posibilidades para pensar la diversidad en la conformación social contemporánea y crear espacios de aprendizaje del respeto y la inclusión.

Asimismo, entendemos el parentesco como una especie de hacer que no refleja una estructura previa, sino que sólo puede entenderse como una práctica que se lleva a cabo. ${ }^{17}$ Esta práctica cubre ciertas necesidades fundamentales y constitutivas para el sujeto humano: capacidad de cuidado, de contacto, de apego, como también, descentramiento de las/os adultas/os para reconocer

11. BUTLER, Judith. «Críticamente subversiva» (1993), en Sexualidades transgresoras. Una antología de estudios queer, Barcelona, Icaria Editorial, s.a. 2002.

12. Ver: Fuss, Diana (ed.), Incide/Out: Lesbian Theories, Gay Theoris, Nueva York, Routledge, 1991; De Lauretis, Teresa. The Practice of Love: Lesbian Sexuality and Perverse Desire, Bloomington, Indiana University, 1994; WiLton, Tamsin, Lesbian Studies: Setting and Agenda, Nueva York, Routledge, 1995 y Nicholson, Linda (ed.), The Second Wave. A Reader in Feminist Theory, Nueva York, Routledge, 1997.

13. Etnografías como las de Malinowski (1913; 1922; 1927; 1929), tantas veces denostadas por su anclaje funcionalista, fueron las que abrieron nuevas perspectivas al mostrar la preeminencia de una paternidad social sobre la biológica en un sistema matrilineal que no reconocía el papel genético del progenitor, lo cual posibilitó, por ejemplo, una desvinculación de lo genético y lo social en el estudio del parentesco (Sánchez Molina; Tomé Martín; Valencia; 2009: 23-24)

14. CASTORIADIS, Cornelius. La institución imaginaria de la sociedad, vol I, Barcelona, Tusquets, 1975. «Imaginario e imaginación en la encrucijada» (1983), en Castoriadis, Cornelius: Figuras de lo pensable. Madrid, Cátedra, 1999, pp. 92-112.

15. FOUCAUlT, Michel. La arqueología del saber (1969), Madrid, Siglo XXI, 1978. Microfísica del Poder. Madrid, La Piqueta, 1978.

16. CASTELAR, Andrés Felipe. «Familia y homoparentalidad: una revisión del tema», en CS No.5, pp. 45-70, enero - junio 2010. Cali - Colombia.

17. BuTLER, Judith. Undoing gender. Op. cit., p. 23.

Feminismo/s 19, junio 2012, pp. 51-65 
a un otro e instituirlo como hija/o. Se trata del lugar en que se posicionan las/os integrantes de la pareja para propiciar el acceso de la cría humana a la cultura, más allá de géneros, «estados civiles», consanguinidades, etc. Sabemos que estas capacidades filiatorias pueden hallarse tanto en una familia homosexual como en una heterosexual, reconociendo el potencial de todo ser humano para libidinizar a un semejante y ocuparse de él.

\section{Familias homoparentales: ¿repetición y/ o diferencia?}

Las familias homoparentales implican en su origen un quiebre a lo instituido, a los roles y funciones establecidos históricamente entre y al interior de los géneros, en tanto posibilitan la transformación del esquema heterocéntrico tradicional, poniendo en cuestión el vínculo naturalizado entre consanguinidad y parentesco ${ }^{18}$

En este apartado nos interesa formular algunos interrogantes en torno a las posibles diferencias y/o repeticiones que estas nuevas familias producen respecto de lo instituido, sea respecto del rol del Estado en la regulación de las relaciones íntimas, sea en la resignificación o no de roles y funciones que históricamente caracterizaron la institución familiar tradicional.

En relación al deseo de tener hijas/os, las familias homoparentales destituyen la tríada familiar del esquema tradicional -madre-padre-niña/o-. De este modo, la «función padre» y la «función madre» deben ser interrogadas más allá de la biología.

Hay que trabajar sobre los prejuicios que responden, en realidad, a la normatividad heterosexual compulsiva, a la familia conyugal, mamá - papá - nene, la concepción tradicional de la familia burguesa. La nueva ley de matrimonio desafía la norma heterosexual, es una infracción al manual de sexualidad y de procreación ${ }^{19}$

Por otra parte, es necesario situar que cuando hablamos de familia no aludimos sólo al reducido núcleo formado por una pareja de adultas y sus hijas/ os, sino que englobamos también a todo no familiar -persona, institución, grupo- que produce efectos de función familia en la cultura. En este sentido, algunos autores se preguntan si acaso el rol del Estado en la regulación de las relaciones íntimas no constituye un nuevo factor de discriminación para aquellas configuraciones vinculares que excedan la nueva normativa del matrimonio igualitario. En términos del análisis que realiza Butler:

18. Castelar, Andrés Felipe. Op. Cit., p. 48.

19. <http://www.pts.org.ar/spip.php?article15792> consultado el 30 de octubre de 2011.

Feminismo/s 19, junio 2012, pp. 51-65 
Se plantea la pregunta de si el giro hacia el matrimonio hace más difícil la discusión en favor de la viabilidad de convenios de parentesco alternativos o en favor del bienestar del «hijo o hija» en una gran cantidad de formas sociales ${ }^{20}$

O como profundizaría Michael Warner ${ }^{21}$ :

... si el impulso para volverse reconocibles dentro de las normas de legitimidad existentes requiere que suscribamos una práctica que deslegitima las vidas sexuales que se estructuran fuera de los lazos del matrimonio y de las suposiciones de la monogamia. ¿La comunidad queer está dispuesta a negar esto? ¿Cuáles serán las consecuencias sociales? ¿Cómo es posible que demos al Estado el poder de reconocernos cuando insistimos en que, sin él, somos irreales e ilegítimos/as? ¿Existen otros recursos que pueden volvernos reconocibles o que nos permiten movilizarnos para desafiar a los regímenes existentes en cuyo interior se encuentran los términos de la posibilidad de ser reconocidos y reconocidas? ${ }^{22}$

La pregunta que nos surge es: ¿existen sujetos legítimos e ilegítimos para filiar a un/a niña/o a la cultura? Es común escuchar que los padres y madres son responsables exclusivos de la conformación de la identidad de la niña/o, estando las parejas homosexuales bajo la lupa, debido a la desconfianza, el desconocimiento, el temor y la incertidumbre social respecto a cómo afectará la orientación sexual de sus padres/madres el desarrollo de las/os hijas/ os. Sin embargo, lamentamos no encontrar el mismo interés en responder la pregunta por las elevadas estadísticas de violencia de género, abuso sexual infantil, maltrato infantil, incesto, etc. en el marco de las tradicionales familias heterosexuales.

Nuevamente Castelar ${ }^{23}$ nos relanza la pregunta mediante una cita del trabajo de Garnets \& Douglas:

...el fenómeno de padres y madres abiertamente homosexuales que crían hijos es una innovación sociocultural única en la era histórica actual; como tal, despierta preguntas sobre el impacto cultural que causa en los niños ${ }^{24}$.

Entendemos que esta es la vía por donde sostener la potencia instituyente de este acontecimiento cultural, es decir, rastrear el impacto que el mismo produce en las instituciones del Estado y en la cotidianidad de los lazos sociales.

20. BUTLER, Judith. Undoing gender. Op. cit. p. 7.

21. WARNER, Michael.«Beyond Gay Marriage», en Wendy Brown y Janet Halley (eds.), Left Legalism/Left Critique, Duke Universtiy Press, Durham, 2010.

22. BUTLER, Judith. Undoing gender. Op. cit. p. 18.

23. Castelar, Andrés Felipe. Op. cit., p. 47.

24. Garnets, Linda. Douglas, Kimmel. Psychological Perspectives on LGB Experiencies, Nueva York. Columbian UP, 2003, p. 498. 
Frente al argumento que advierte de la «peligrosidad» de los vínculos homoeróticos para la crianza de las/os niñas/os, recordamos nuevamente que la conformación de la identidad del hijo/a es un proceso complejo que incluye no sólo a los padres, las madres sino a educadoras/es y diferentes modelos del espectro social. Dice Silvia Bleichmar ${ }^{25}$ que:

... la identificación remite a las formas de transmisión con las cuales el adulto proporciona ordenadores, moldes, formas de coagulación del ser y de las instancias de prohibición y que una cuestión básica de esta identificación es la humanización del otrx, como pertenencia a la especie. Estos efectos identificatorios en el hijx se apoyan en factores intersubjetivos, grupales y sociales internalizados que complejizan aún más el proceso. Siendo preciso incluir estos otros cortes extra-familiares que atraviesan y sobrepasan a la familia y que también intervienen en la conformación de la identidad de lxs niñxs.

En este mismo sentido, coincidimos con Castelar ${ }^{26}$ en que la legitimación del matrimonio homosexual y de la homoparentalidad transformará a futuro el concepto de familia, así como posibilita la transformación del esquema heterocéntrico tradicional y desafía el vínculo naturalizado entre consanguinidad y parentesco.

\section{Movimientos sociales y producción de derechos}

En materia de Derechos Humanos, sabemos que quien los viola es el Estado y sus funcionarios. Por tanto se nos plantea una paradoja al instar a este mismo actor social a que reconozca los derechos de aquellos sujetos subalternos al orden cultural y político hegemónico. En esta paradoja se sostienen las organizaciones de Derechos Humanos y los movimientos sociales, en ese campo limítrofe entre lo legal y lo legítimo, ampliando los horizontes de legitimidad a través de históricas luchas, que como sabemos han costado y siguen costando muchas vidas.

Experiencias como la cubana nos informan acerca de la enorme transformación cultural y política que requiere el respeto por la diversidad sexual. En palabras de la directora del Centro Nacional de Educación Sexual (CENESEX) de Cuba, Mariela Castro Espín ${ }^{27}$ :

25. BLEICHMAR, Silvia. «Las nuevas cuestiones ponen en crisis viejas teorías» (entrevista de Eva Rotenberg a Silvia Bleichmar), En: Homoparentalidades. Nuevas familias. Bs. As. Lugar Editorial. 2007, pp. 91- 98.

26. CASTELAR, Andrés Felipe. Op. cit., p. 48.

27. <http://www.juventudrebelde.cu/cuba/2010-06-09/mariela-castro-espin-se-entrevistara-con-los-lectores-de-juventud-rebelde/>, consultado el 20 de enero de 2012. 
... el género, la orientación sexual, la identidad y el rol de género, son categorías de análisis de realidades humanas no desviadas, ni inmorales, ni anormales, ni perversas, ni diabólicas. Históricamente han sido utilizadas para controlar a las personas y los grupos humanos por parte de los grupos de poder económicamente dominantes en las sociedades clasistas y se han impuesto como patrones de normatividad. Las experiencias socialistas que se propusieron establecer sociedades más justas, reprodujeron esos mismos esquemas de pensamiento para la regulación social. Por eso, desde la experiencia socialista cubana estamos tratando de esclarecer el origen de todas estas creencias que conducen al estigma y la violación de derechos, para que seamos capaces de superar estos atavismos que no nos permiten como sociedad justa e inclusiva.

Por su parte, el movimiento feminista ha contribuido a la conquista de derechos impulsando importantes cambios culturales a través de la producción de conocimientos, asentados en metodologías específicas de investigación que subvierten paradigmas hegemónicos en $\mathrm{Ciencia}^{28}$, así como a través de las incesantes movilizaciones en las calles. La noción de género sexual desarrollada a partir de los años 70' y que representa en su origen una derivación de los estudios de la mujer, ha constituido una herramienta fundamental para visibilizar los órdenes morales y de dominación de la sexualidad y sus representaciones masculinas y femeninas. En términos de Mérida Jiménez:

La consolidación de un paradigma y la creación de una genealogía contrapuesta al orden patriarcal como mecanismo de identificación de una historiografía de las mujeres propició que cuando algunos miembros de los grupos reivindicativos de los derechos homosexuales intentaran trazar su árbol genealógico acudieran al feminismo como el mejor de los referentes posibles, dada la proyectabilidad de sus resultados ${ }^{29}$.

La vulneración de derechos constituye un atentado a los procesos de subjetivación humana, y ello no precisamente por parte de parejas del mismo sexo que amorosamente desean criar hijas/os, sino por parte de complejos entramados institucionales y de poder que expulsan del orden cultural a un sector de la sociedad.

Antes que promover o imponer el modelo de familia tradicional, monógamo, estandarizado y distribuido por roles en función de la diferencia sexual, el derecho al matrimonio implica contar con la posibilidad de conformar jurídicamente una estructura de tipo familiar en igualdad de condiciones legales,

28. HaraWAY, Donna. Ciencia, cyborgs y mujeres. La reinvención de la naturaleza. Madrid, Cátedra 1995.

29. MÉRIdA Gimenez, Rafael M. (ed.), «Prólogo», Sexualidades transgresoras. Una antología de estudios queer. Barcelona, Icaria editorial, 2002. 
con la firme intención de eliminar (o al menos reducir) la injusticia de esta exclusión. ${ }^{30}$

En cuanto al rol del Estado, es ineludible decir que han sido las históricas luchas sociales las que han introducido importantes cambios en la ley, sin ellas es inconcebible pensar en las transformaciones culturales. Asimismo, quienes crean que una vez plasmadas en la ley las diversas realidades sexuales, entre otras, se acaban las injusticias, sin duda están errando. Como ya lo hemos planteado en otros trabajos, la continuidad de las luchas sociales es indispensable, tanto para la apropiación de esos cambios por los mismos protagonistas, cuanto para la asunción de estos por la sociedad en su conjunto ${ }^{31}$

\section{Conclusiones}

Consideramos que la sanción de la Ley de Matrimonio Igualitario resulta una herramienta trascendente para la visibilización y legitimación de las nuevas configuraciones familiares vinculadas a la diversidad sexual. Sin embargo, reafirmamos paralelamente el papel trascendental de las luchas de los movimientos sociales - de derechos humanos y de diversidad sexual- en la instalación en la agenda pública de las demandas de estas familias y grupos, así como en su contribución a la apropiación de las modificaciones sociales por parte de los protagonistas y del resto de la sociedad, constituyendo un verdadero motor de transformaciones culturales.

En efecto, estas nuevas configuraciones familiares, modifican profundos patrones de creencias en relación al parentesco y la filiación, entre muchos otros, desafiando los saberes instalados por la psicología, la biología y la sociología. En este sentido, se requiere una revisión y reelaboración de categorías y conceptos que permitan cuestionar los binarismos que han permeado la comprensión de la realidad. Si situamos el basamento de la relación materno/ filial o paterno/filial exclusivamente en la biología, dejamos fuera la posibilidad de comprensión de estos vínculos que constituyen parte fundamental de las transformaciones sociales y culturales acaecidas en nuestra sociedad, siendo preciso tener en cuenta el deseo y la voluntad como fundamentos del vínculo filiatorio.

30. Castelar, Andrés Felipe. Op. cit., p. 50.

31. Buzaglo, Analía; Capella, Laura. «Procesos de subjetivación y horizontes de legitimidad» (2011), III Congreso Argentino - Latinoamericano de Derechos Humanos: repensar la Universidad en la diversidad Latinoamericana, Facultad de Humanidades y Artes, UNR, Rosario, Argentina. Dirección de Derechos Humanos de la Subsecretaría de Cultura de la UNR días 3, 4, 5 y 6 de mayo de 2011. 
Por otro lado, es preciso incluir el amplio entramado que participa en la crianza de las/os niñas/os y que excede a las figuras de madres y padres, instalando significados influyentes en su desarrollo.

Las miradas biologicistas influyen, también, en las posiciones que algunas disciplinas como el Psicoanálisis sostienen acerca de la cuestión edípica -aún cuando las relecturas de los textos clásicos hayan ya echado luz sobre el tema-. En este sentido, es preciso insistir en repensar las conceptualizaciones que asocian mecánicamente las funciones maternas y paternas a los sexos femenino y masculino respectivamente, considerando que alrededor de la «función de corte» y del incesto, el escenario de la novela edípica puede ser ampliado y resignificado. Desde ahí nos preguntamos: si dichas funciones pueden ser ejercidas indistintamente por cualquiera de los géneros/sexos implicados, ¿qué nuevos significados e implicaciones trae aparejado este hecho para la constitución identitaria de las/os niñas/os? Y también: ¿qué nuevas palabras y discursos advienen a partir de estas realidades que «irrumpen» como acontecimiento y que develan y se rebelan en un 'cuerpo social' y familiar en construcción y deconstrucción, como proceso de creación de identidades y de subjetividades cambiantes?

La historia de las sexualidades, de todos aquellas/os que desde movimientos reivindicativos y visibilizadores, de lo que durante mucho tiempo ha debido permanecer oculto y reservado al terreno de lo privado, nos muestra un proceso de desvelamiento. Pensar que es sólo «cultural» en su concepción más limitante del término, sería definirlo políticamente de forma sesgada e insistir en excluir.

La homoparentalidad nos interpela en y desde los múltiples atravesamientos que «tocan» a las Ciencias Sociales en sus disciplinas, teorías e intervenciones; a lo Político -como el poder que regula cuerpos, acciones y relaciones- $y$ al rol de los Estados en la procura de leyes que reflejen y legitimen estas realidades, ampliando realmente el universo de derechos legales, económicos, en definitiva, vitales. Estas familias interpelan a los roles y funciones conformadas desde un heterocentrismo naturalizado y los nutren, a su vez, de nuevas y creativas formas de construcción: de subjetividades, de formas de encontrarse y de hacer núcleos que cobijan, protegen y crían hijas/os.

Si pensamos al acontecimiento como advenimiento, como irrupción de algo que «aparece» y que irrumpe e interrumpe un orden dado, es necesario hacerlo historizando los discursos que lo mantuvieron oculto -así como la historia de aquellas/os que tanto desde lo privado, lo público-político, lo social y cultural, han trabajado desde los intersticios de las hegemonías. 
Sostenemos además, desde las diferentes prácticas profesionales en ámbitos públicos y privados, la importancia de la puesta en juego de discursos inclusivos que operen como habilitadores del despliegue de estas nuevas realidades. Tanto en intercambios académicos, cuanto en espacios de reflexión sobre nuestros abordajes clínicos, de intervención y/o asistencia, observamos la internalización, naturalización y el sostenimiento de un andamiaje discursivo determinado por y desde la hegemonía heteronormativa. Ya sea en los servicios de salud, donde constatamos que se invisibilizan mediante ocultamientos y silencios las problemáticas que atraviesa la diversidad sexual, o en espacios educativos, donde la presencia de niñas/os con dos papás ó dos mamás aún no está instalada entre las temáticas relativas a «la familia»; consideramos que las realidades emergentes deben encontrar un lugar de plena integración en los diferentes espacios por los que transita la cotidianeidad. Sin embargo, es evidente la persistencia de leyes, normas y reglamentos laborales que aún no contemplan beneficios sociales para parejas y/o familias de diversidad sexual, restando aún un largo camino para lograr que sus derechos sean reconocidos y ejercidos como los de las/os demás ciudadanas/os.

Por último, quisiéramos retomar aquellos posibles aportes que estas nuevas configuraciones familiares podrían producir en el interior de las estructuras tradicionales del matrimonio y el parentesco, tal como éstas han sido concebidas en nuestras sociedades occidentales.

Si consideramos que el patriarcado ha sido una estructura de dominación que ha permeado gran parte de la historia de la institución matrimonial, con el consecuente sojuzgamiento de la mujer, es válido preguntarnos por las posibles redefiniciones de la distribución del poder que puede producirse en parejas y familias homoparentales. En efecto, éstas desafían la idea de una asignación de roles basada en características biológicas supuestamente naturales, quedando la distribución del poder y las actividades cotidianas a merced exclusiva de acuerdos y negociaciones entre las/os involucradas/os. Si ya no existen géneros dicotómicos con funciones socialmente establecidas: ¿cuáles serán los criterios sobre los que se asiente la distribución de tareas? ¿Supondrá ello una mayor democratización de las mismas?

Si bien creemos que la pareja homoparental no garantiza la no reproducción de posiciones desiguales de poder, resaltamos que todo movimiento en las estructuras puede habilitar posibilidades de redefinición de las relaciones de fuerza naturalizadas. En este sentido nos preguntamos si el matrimonio puede ser reapropiado y resignificado como herramienta capaz de instituir significados diferentes a aquellos sobre los cuales fue creado, es decir, como 
herramienta de ampliación de derechos de ciudadanía para determinados grupos históricamente postergados.

En relación al parentesco, nos preguntamos sobre qué otros fundamentos se reescribirán los vínculos filiatorios entre padres/madres e hijas/os, ahora que la biología no resulta el único argumento válido para justificar y fundamentar los vínculos entre padres/madres e hijas/os. Y además: ¿qué sentidos imaginarios construirán las/os niñas/os para significar estas nuevas vincularidades en el marco de las nuevas escenas familiares?

Confiamos en que un ejercicio permanente en la invención de respuestas a estas preguntas, redundará en la posibilidad de generar espacios y mecanismos sociales y legales tendientes a hacer más democráticas e inclusivas las sociedades por las que transitamos cotidianamente a lo largo de nuestra vida.

\section{Referencias bibliográficas}

BLEICHMAR, Silvia. «Las nuevas cuestiones ponen en crisis viejas teorías» (entrevista de Eva Rotenberg a Silvia Bleichmar). En: Homoparentalidades. Nuevas familias. Bs. As. Lugar Editorial. 2007. pp. 91-98.

ButLer, Judith (2002) El género en disputa. Trad. Paidós - UNAM, México DF (original de 1990).

- (2004) Undoing Gender, Routledge, Nueva York, 2004.

BuZAGLO, Analía; CAPELLA, Laura. «Procesos de subjetivación y horizontes de legitimidad», trabajo presentado en el III Congreso Argentino - Latinoamericano de Derechos Humanos: repensar la Universidad en la diversidad Latinoamericana, Facultad de Humanidades y Artes, de la UNR Rosario, Argentina. Dirección de Derechos Humanos de la Subsecretaría de Cultura de la UNR días 3, 4, 5 y 6 de mayo de 2011.

CASTELAR, Andrés Felipe. La identidad en disputa: una propuesta filosófica de Judith Butler. Tesis de magister en filosofía. Dirección de Gabriela Castellanos, PhD. Facultad de Humanidades, Universidad del Valle, Cali. 2008

- «Familia y homoparentalidad: una revisión del tema» En Cs No.5, pp. 45-70, enero - junio 2010. Cali - Colombia.

CASTRO Espín, Mariela. Directora del Centro Nacional de Educación Sexual (CENESEX) http://www.juventudrebelde.cu/cuba/2010-06-09/mariela-castro-espinse-entrevistara-con-los-lectores-de-juventud-rebelde/

ERIBON, Didier. Reflexiones sobre la cuestión gay. Anagrama, Barcelona. 1999

FASSIN, Eric. «Same Sex, Different Politics: 'Gay Marriage' Debates in France and the United States», Public Culture, vol. 13, núm. 2 (primavera), (2001) pp. 215-232.

Garnets, Linda. Kimmel, Douglas. Psychological Perspectives on LGB Experiencies. Columbian UP: NY. 2003. 
HaraWAY, Donna. Ciencia, cyborgs y mujeres. La reinvención de la naturaleza. Madrid: Cátedra (1995).

KONTANTINOVSKY, Verónica. «Familias Homoparentales: Testimonio y propuestas para una intervención en el campo. La salida del clóset y una revisión de los prejuicios a la hora de atender a familias homoparentales». En Revista Enfoque Gestáltico N²3, (2011).

MAFFIA, Diana. «Familia y adopción: reflexiones acerca de la homoparentalidad». En: Rotenberg, E., Wainer Agrest, B. (comp.): Homoparentalidades. Nuevas familias. Bs. As. Lugar Editorial. 2007. pp. 57-62.

MÉRIDA JimÉnEZ, Rafael M. (ed.), «Prólogo», Sexualidades transgresoras. Una antología de estudios queer. Barcelona: Icaria editorial. 2002.

ROUDINESCO, Elisabeth. La familia en desorden. FCE, Bs. As.2002.

SÁnchez Molina, Raúl; Tomé Martín, Pedro y Valencia, Ma Ángeles. «Nuevos tiempos, nuevas familias: aproximaciones etnográficas en el estudio de configuraciones familiares contemporáneas» En Revista Latinoamericana de Estudios de Familia Vol. 1, enero - diciembre, (2009). pp. 22-45.

SEGAL, Hanna. «Hanna Segal interviewed by Jacqueline Rose», Women: A Cultural Review, vol. 1, núm. 2, (1990), pp.198-214.

Stone, Linda. «Gay Marriage and Anthropology». Anthropology News 45, N 5: 10. (2004), pp 3-60.

VolNOVICH, Juan Carlos, «Adopción: un derecho dentro del matrimonio igualitario», entrevista publicada el día 5 de agosto de 2010 en: http://www.pts.org. ar/spip.php?article15792.

WARnER, Michael. «Beyond Gay Marriage», en Wendy Brown y Janet Halley (eds.), Left Legalism/Left Critique, Duke Universtiy Press, Durham. 2002. 